\title{
Planetary nebula abundance determinations: A view from 1D-RHD simulations
}

\author{
Ralf Jacob, Christer Sandin, Detlef Schönberner \\ and Matthias Steffen \\ Leibniz-Institut für Astrophysik Potsdam, \\ An der Sternwarte 16, D-14482 Potsdam, Germany \\ email: rjacob@aip.de
}

\begin{abstract}
In the last years (metallicity-dependent) radiation-hydrodynamics simulations have become a powerful tool to understand the formation and evolution of PNe in terms of simple morphologies and kinematics. Contrary to photoionization models, with their ad-hoc assumptions on structure and physics, the RHD models are self-consistent with respect to their density distribution, velocity field, chemical composition, and stellar evolution. We use our models as simple proxies for real PNe and investigate the reliability of common abundance determination methods, which are based on either plasma diagnostics or static photoionization (PI) models.
\end{abstract}

Keywords. hydrodynamics, planetary nebulae: general, ISM: abundances

\section{1. (Non-)compliance to thermal and ionization equilibrium}

Nebular gas is heated by ionization and cooled by line radiation. Additionally, the gas is subject to dynamical processes: cooling (heating) by expansion (compression). PNe expand the faster the lower their metallicity, $Z$ ! Already at $0.5-1.0$ dex below the typical Galactic disk (GD) composition expansion cooling contributes significantly to the total cooling, since the line-cooling efficiency drops simultaneously with $Z$ - a fact generally neglected in static PI-codes (Schönberner et al. 2010). Hence, incorrectly attributed electron temperatures lead, e.g., to incorrect strengths of collisionally excited lines, depending on the temperature sensitivity of the corresponding wavelength range. Therefore, abundances of PNe with high expansion velocities, such as metal-poor PNe or certain bipolar PNe, should be treated with care (Sandin et al. 2010)! Ionization equilibrium (I-EQ) is in general well established in the main nebular body. Deviations for main ionization stages (typically $<10 \%$ ) are due to different collisional ionization rates according to different temperatures. The low-density halos are always far off I-EQ (Schönberner et al. 2010).

\section{Influence of morphology or Is the sulfur anomaly really for real?}

Plasma diagnostics has the disadvantage that unseen ionization stages must be corrected for with ionization correction factors (ICF) - again based on PI-models! The detailed PN density distribution has strong impact on its ionization structure. For instance, the sulfur anomaly, as first coined by Henry et al. (2004), describes the apparent deficiency of sulfur in $\mathrm{PNe}$ relative to $\mathrm{H}$ II-regions. According to nucleosynthesis theory both should follow the same narrow correlation. The original conjecture of incorrect S-ICFs got out of sight with the "confirmation" of the S-anomaly through direct IR observations (e.g. Shaw et al. 2010). However, the search for an additional sink of sulfur in PNe has failed so far (cf. Henry et al., this volume). We argue that $\mathrm{S}^{4+}$ is this sink, since its contribution is severely underestimated in (over)simplified PI-models with uniform density, as those of the ICFcomputation of Kwitter \& Henry (2001) and Henry et al. (2004) with CLOUDY94. 


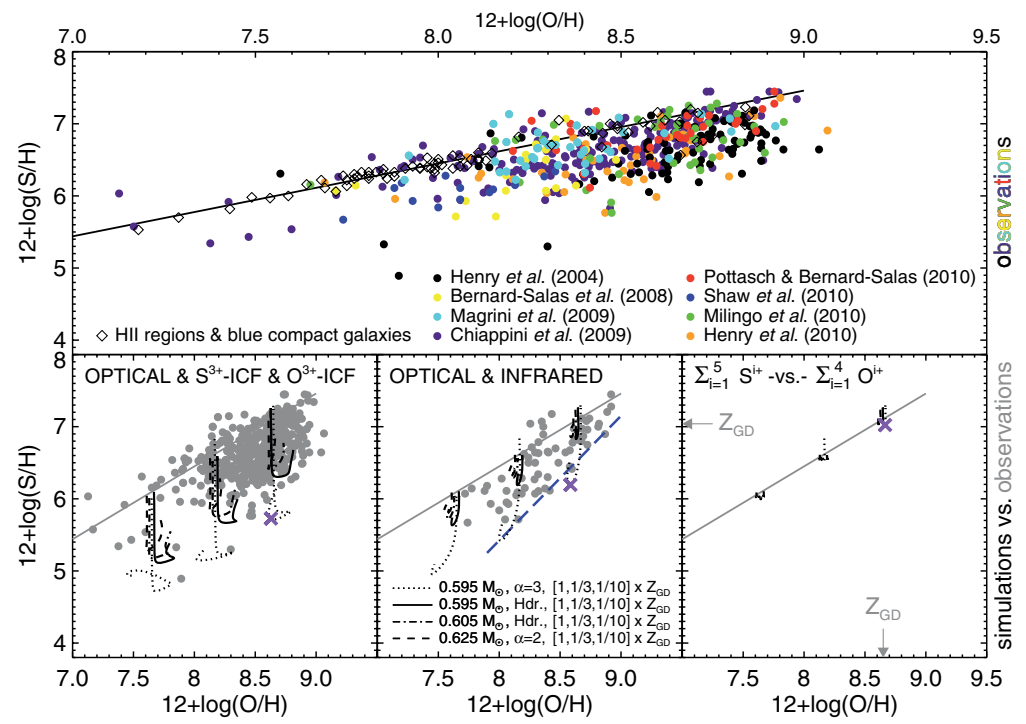

Figure 1. Top: PN data collected from the literature for the Milky Way, LMC, SMC, \& M33. The solid line is the fit to the narrow correlation defined by HII regions and BCGs. Bottom: The first two panels split the sample into optical studies corrected with ICFs and into the ones based on additional IR data (w/o the need for ICFs). Both are compared with 1D-RHD sequences (evolving from top to bottom). The combined IR-based sample indicates an increasing S-deficiency with decreasing $Z$. Our models predict, that faster expanding low- $Z \mathrm{PNe}$ experience higher dilutions, favoring thus higher degrees of ionization $\left(\mathrm{S}^{4+}\right.$ even becomes the main ionization stage). The $\mathrm{S}$-anomaly vanishes if the next higher ionic species $\left(\mathrm{S}^{4+}, \mathrm{O}^{4+}\right.$, poss. $\left.\mathrm{S}^{5+}\right)$, are also included (last panel)! X marks the an evolved model used to compare with CLOUDY (Fig. 2).

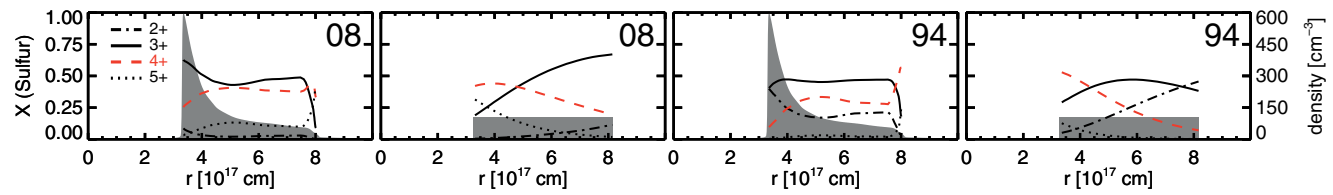

Figure 2. Variation of ionization fractions for cases of variable vs. uniform density from CLOUDY (c08.01 vs. c94, last described in Ferland et al. 1998) The exemplary RHD-model density structure (with $Z=Z_{\mathrm{GD}}$ and $\mathrm{CSPN} @ T_{\text {eff }}=123.000 \mathrm{~K} \& L=3800 \mathrm{~L}_{\odot}$; the $\mathrm{X}$ in Fig. 1) and its volume-averaged (!) mean-density counterpart are visualized in gray shades. Recent improvements to the atomic data of sulfur will also alter the sulfur-ICFs. Nevertheless, the discrepancy due to different morphologies stays a general problem!

\section{References}

Bernard-Salas, J., Pottasch, S. R., Gutenkunst, S., et al. 2008, ApJ, 672, 274

Chiappini, C., Górny, S. K., Stasińska, G., \& Barbuy, B. 2009, A\& A, 494, 591

Ferland, G. J., Korista, K. T., Verner, D. A., et al. 1998, PASP, 110, 761

Kwitter, K. B. \& Henry, R. B. C. 2001, ApJ, 562, 804

Henry, R. B. C., Kwitter, K. B., \& Balick, B. 2004, AJ, 127, 2284

Henry, R. B. C., Kwitter, K. B., Jaskot, A. E., et al. 2010, ApJ, 724, 748

Magrini, L., Stanghellini, L., \& Villaver, E. 2009, ApJ, 696729

Milingo, J. B., Kwitter, K. B., Henry, R. B. C., \& Souza, S. P. 2010, ApJ, 711, 619

Pottasch, S. R. \& Bernard-Salas, J. 2010, A\&A, 517, 95

Sandin, C., Jacob, R., Schönberner, D., Steffen, M., \& Roth, M. M. 2010, A\&AA, 512, A18

Schönberner, D., Jacob, R., Sandin, C., \& Steffen, M. 2010, A\&A, 523, A86

Shaw, R. A., Lee, T.-H., Stanghellini, L., et al. 2010, ApJ, 717, 562 